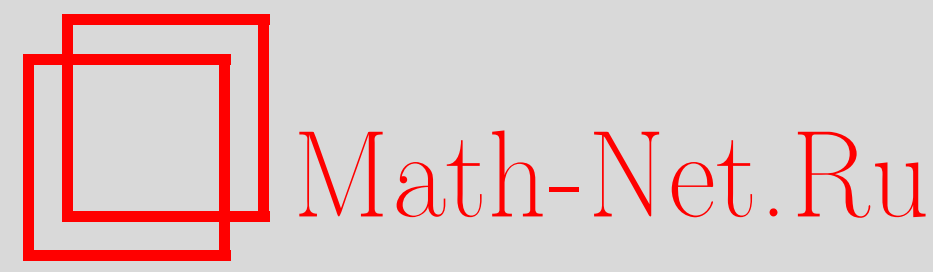

В. С. Климов, О топологических характеристиках негладких функционалов, Изв. РАН. Сер. матем., 1998, том 62, выпуск 5, 117-134

DOI: https://doi.org/10.4213/im212

Использование Общероссийского математического портала Math-Net.Ru подразумевает, что вы прочитали и согласны с пользовательским соглашением http://www . mathnet.ru/rus/agreement

Параметры загрузки:

IP : 54.162 .127 .20

26 апреля 2023 г., 12:24:43 
УДК 517.946

\author{
В. С. Климов
}

\title{
О топологических характеристиках негладких функционалов
}

\begin{abstract}
Устанавливаются бесконечномерные варианты теоремы Пуанкаре-Хопфа для многозначных векторных полей, порождаемых операторами монотонного типа. Приводятся условия стабилизации групп гомологий замкнутых подмножеств банахова пространства при аппроксимации конечномерными сечениями. Основное внимание уделяется изучению топологических характеристик лебеговых множеств липшицевых функционалов, определенных на замкнутом выпуклом подмножестве рефлексивного пространства.

Библиография: 26 наименований.
\end{abstract}

В статье приводятся условия стабилизации групп гомологий компактов, возникающих при аппроксимации ограниченного замкнутого подмножества $B$-пространства его конечномерными сечениями. Устанавливаются варианты теоремы Пуанкаре-Хопфа для бесконечномерных многозначных векторных полей, порождаемых операторами монотонного типа [1]-[3]. Основное внимание уделяется изучению топологических характеристик нижних лебеговых множеств липшицевых функционалов, определенных на выпуклом замкнутом подмножестве рефлексивного пространства.

Важную роль в работе играют геометрические методы нелинейного анализа [4]-[9]. Близким вопросам посвящены публикации [10]-[16]. Теоремы об индексе множества точек минимума нашли приложения к оценкам числа решений краевых задач, возникающих в нелинейной теории оболочек, химической кинетике и теории сверхпроводимости Гинзбурга-Ландау (см., например, [2, с. 155-158], [10], [24]).

Основные обозначения: $\mathscr{B}(\mathfrak{R})(\mathscr{K}(\mathfrak{R}))$ - совокупность ограниченных (компактных) подмножеств метрического пространства $\mathfrak{R}, H_{l}(\mathfrak{R}), l=0,1, \ldots,-$ группы целочисленных гомологий с компактными носителями пространства $\mathfrak{R}$ (см. [17, гл. 9], $[18$, c. 316] $), b_{l}(\Re)$ - ранг группы $H_{l}(\Re)$, называемый $l$-м числом Бетти пространства $\mathfrak{R}$,

$$
\mathrm{X}(\mathfrak{R})=b_{0}(\mathfrak{R})-b_{1}(\mathfrak{R})+\cdots+(-1)^{l} b_{l}(\mathfrak{R})+\cdots
$$

- эйлерова характеристика пространства $\mathfrak{R}$ (это определение имеет смысл лишь в случае, когда $b_{l}(\mathfrak{R})<\infty \quad \forall l$ и $b_{l}(\mathfrak{R})=0$ при $\left.l \geqslant l_{0}\right) ; \overline{\mathfrak{M}}\left(\mathfrak{M}^{0}, \partial \mathfrak{M}\right)$ - замыкание (внутренность, граница) множества $\mathfrak{M} \subset \mathfrak{R}, d_{\mathfrak{R}}(x, \mathfrak{M})=\inf \{\rho(x, y), y \in \mathfrak{M}\}$ - pacстояние в метрике $\rho$ пространства $\mathfrak{R}$ от элемента $x$ до множества $\mathfrak{M} ; \theta_{\mathfrak{R}}(\mathfrak{N}, \mathfrak{M})=$ $\sup \left\{d_{\mathfrak{R}}(x, \mathfrak{M}), x \in \mathfrak{N}\right\}$ - уклонение множества $\mathfrak{N} \subset \mathfrak{R}$ от множества $\mathfrak{M} \subset \mathfrak{R} ;$ $\Gamma(X)$ - совокупность конечномерных подпространств банахова пространства $X$, $X^{*}$ - сопряженное к $X$ пространство, $\langle\cdot, \cdot\rangle$ - каноническая билинейная форма на

(C) в.С. Климов 1998 
$X \times X^{*}, \sigma\left(x^{*}, \mathscr{M}\right)=\sup \left\{\left\langle x, x^{*}\right\rangle, x \in \mathscr{M}\right\}\left(x^{*} \in X^{*}\right)$ - опорная функция множества $\mathscr{M} \subset X, \operatorname{Cv}(X)$ - совокупность всех непустых выпуклых замкнутых подмножеств пространства $X$. Если $C \in \operatorname{Cv}(X), x \in C$, то определяемые соотношениями

$$
T_{C}(x)=\overline{\bigcup_{h>0} h(C-x)}, \quad N_{C}(x)=\left\{x^{*} \in X^{*},\left\langle v-x, x^{*}\right\rangle \leqslant 0 \quad \forall v \in C\right\}
$$

множества называют касательным и нормальным конусами $к$ множеству $C$ в точке $x$. Через $\Lambda(\mathscr{M}, Y)$ обозначается совокупность отображений множества $\mathscr{M} \subset X$ в банахово пространство $Y$, удовлетворяющих локальному условию Липшица, т.е. $F \in \Lambda(\mathscr{M}, Y)$, если для каждой точки $x$ из $\mathscr{M}$ найдутся такая ее окрестность $O$ и такая константа $L$, что

$$
\left\|F\left(x_{1}\right)-F\left(x_{2}\right)\right\| \leqslant L\left\|x_{1}-x_{2}\right\|, \quad x_{i} \in O, \quad i=1,2
$$

здесь и далее имеются в виду относительные окрестности точки $x . \mathrm{Kласс} \Lambda(\mathscr{M}, \mathbb{R})$ действительных на $\mathscr{M}$ функщий, удовлетворяюших локальному условию Липшица, обозначается символом $\Lambda(\mathscr{M})$.

Многозначное отображение множества $\mathscr{M}_{1}$ во множество $\mathscr{M}_{2}$ - это оператор, ставяший в соответствие элементу $x$ из $\mathscr{M}_{1}$ непустое множество $\mathscr{F}(x) \subset \mathscr{M}_{2}$; если $\mathscr{M} \subset \mathscr{M}_{1}$, то $\mathscr{F}(\mathscr{M})=\cup \mathscr{F}(x)(x \in \mathscr{M})$ - область значений отображения $\mathscr{F}$ на множестве $\mathscr{M}$. Отображение $\mathscr{F}: \mathscr{M}_{1} \rightarrow \mathscr{M}_{2}\left(\mathscr{M}_{1}, \mathscr{M}_{2}\right.$ - подмножества метрических пространств $\mathfrak{R}_{1}, \mathfrak{R}_{2}$ соответственно) называют ограниченнылм, если $\mathscr{F}(\mathscr{M}) \in \mathscr{B}\left(\Re_{2}\right)$ для каждого $\mathscr{M}$ из $\mathscr{B}\left(\Re_{1}\right), \mathscr{M} \subset \mathscr{M}_{1}$. Оператор тождественного преобразования обозначается символом $I$. Все рассматриваемые в статье банаховы пространства предполагаются действительными.

1. Всюду далее $X$ - сепарабельное рефлексивное банахово пространство, $\|\cdot\|$ и $\|\cdot\|_{*}-$ нормы в $X$ и сопряженном к нему $X^{*}$, символы $\rightarrow$ и $\rightarrow$ означают слабую и сильную сходимости соответственно; $Q \in \operatorname{Cv}(X), X_{0}=\operatorname{Lin}(Q-Q)$ - линейная оболочка множества $Q-Q$; ниже предполагается, что $\bar{X}_{0}=X$. Обозначим через $\Gamma(Q)$ часть $\Gamma(X)$, состоящую из пространств $E$, для которых внутренность $\operatorname{ri}_{E}(Q \cap E)$ множества $Q \cap E$ относительно пространства $E$ непуста. Отметим некоторые свойства класса $\Gamma(Q)$ :

1) если $E \in \Gamma(Q), z \in Q, \mathscr{H}=\operatorname{Lin}\{E, z\}-$ линейная оболочка $E$ и $z$, то $\mathscr{H} \in \Gamma(Q)$

2) если $E_{0} \in \Gamma(X)$ и $Q \cap E_{0} \neq \varnothing$, то существует пространство $E$ из $\Gamma(Q)$, $E \subset E_{0}$, для которого $Q \cap E=Q \cap E_{0}$;

3 ) найдется последовательность $E_{n}, n=1,2, \ldots$, обладающая свойствами

$$
E_{n} \subset E_{n+1}, \quad E_{n} \in \Gamma(Q), \quad \overline{\bigcup_{n=1}^{\infty}\left(Q \cap E_{n}\right)}=Q
$$

(последовательность $E_{n}$ с подобными свойствами называем далее исчерпьвающей множество $Q$ ). Сушествование исчерпывающих последовательностей легко выводится из сепарабельности пространства $X$. 
Пусть $\mathscr{M}=\overline{\mathscr{M}} \subset Q$. Обозначим через $S(\mathscr{M})$ совокупность ограниченных многозначных операторов $A: \mathscr{M} \rightarrow X^{*}$, удовлетворяющих условиям:

1) $A(x) \in \operatorname{Cv}\left(X^{*}\right) \forall x \in \mathscr{M}$;

2) для произвольных последовательностей $x_{n} \in \mathscr{M}, x_{n}^{*} \in A\left(x_{n}\right)$, обладаюших свойствами

$$
x_{n} \rightarrow x, \quad x_{n}^{*} \rightarrow x^{*}, \quad \varlimsup_{n \rightarrow \infty}\left\langle x_{n}, x_{n}^{*}\right\rangle \leqslant\left\langle x, x^{*}\right\rangle,
$$

имеют место соотношения

$$
x_{n} \rightarrow x, \quad x^{*} \in A(x) .
$$

Первое условие означает выпуклозначность оператора $A$. Оно автоматически выполнено для однозначного отображения. Второе условие означает замкнутость оператора $A$ в некотором усиленном варианте. Близкие классы однозначных операторов рассматривались в [1]-[3], [16]. Примеры многозначных операторов класса $S(\mathscr{M})$ можно найти в [14], [15]. Отметим, что сужение оператора $A$ класса $S(\mathscr{M})$ на замкнутое множество $\mathscr{M}_{0} \subset \mathscr{M}$ есть оператор класса $S\left(\mathscr{M}_{0}\right)$. Оператор класса $S(\mathscr{M})$ хеминепрерывен на $\mathscr{M}$ в следующем смысле: для любого $v$ из $X$ функция $\sigma\left(v_{0} A(\cdot)\right): \mathscr{M} \rightarrow \mathbb{R}$ полунепрерьвна сверху. Сумма оператора класса $S(\mathscr{M})$ и однозначного вполне непрерывного из $\mathscr{M}$ в $X^{*}$ оператора есть оператор класса $S(\mathscr{M})$.

Элемент $x \in \mathscr{M}$ назовем особой точкой оператора $A$ класса $S(\mathscr{M})$, если $0 \in$ $A(x)+N_{Q}(x)$, что равносильно существованию элемента $x^{*}$ из $A(x)$, для которого

$$
\left\langle v-x, x^{*}\right\rangle \geqslant 0 \quad \forall v \in Q
$$

Соотношение (3) означает, что особые точки оператора $A$ являются решениями соответствуюшего вариационного неравенства [19, с. 255]. Для вьполнения (3) достаточно, чтобы имело место соотношение

$$
\left\langle v-x, x^{*}\right\rangle \geqslant 0 \quad \forall v \in Q_{R},
$$

где $R>\|x\|, Q_{R}=\{y \in Q,\|y\| \leqslant R\}$. В случае $x \in Q^{0}$ соотношение (3) равносильно включению $0 \in A(x)$. Оператор $A$ класса $S(\mathscr{M})$ назовем невырожденнылм на множестве $\mathfrak{M} \subset \mathscr{M}$, если $\mathfrak{M}$ не содержит особых точек.

ЛЕмма 1. Пусть оператор $A$ класса $S(Q)$ невырожден на ограниченном замкнутом множестве $\mathfrak{M} \subset Q$. Тогда существуют $E$ из $\Gamma(Q)$, равномерно ограниченное отображсение $w$ класса $\Lambda(Q, X)$ и постоянная $\delta>0$, обладающие свойствами

$$
w(x) \in \operatorname{ri}(Q \cap E) \quad \forall x \in Q, \quad \sigma(w(x)-x, A(x))<-\delta \quad \forall x \in \mathfrak{M} .
$$


ДокАЗАТЕЛЬСТво. Будем считать, что $Q \in \mathscr{B}(X)$. Это предположение не уменьшает обшности, поскольку в противном случае множество $Q$ можно заменить множеством $Q_{R}$, где $R>\sup \{\|x\|, x \in \mathfrak{M}\}$. Фиксируем исчерпьвающую множество $Q$ последовательность $E_{n}(n=1,2, \ldots)$. Положим

$$
\mathfrak{M}_{n}=\left\{x \in Q, d_{X}(x, \mathfrak{M}) \leqslant \frac{1}{n}, \sigma(v-x, A(x)) \geqslant-\frac{1}{n} \quad \forall v \in Q \cap E_{n}\right\} .
$$

Если $x_{n} \in \mathfrak{M}_{n}$ при некотором $n$, то согласно теоремам о минимаксе (см., например, [9]) найдется такой элемент $x_{n}^{*}$ из $A\left(x_{n}\right)$, что

$$
\min _{v \in Q \cap E_{n}}\left\langle v-x_{n}, x_{n}^{*}\right\rangle=\min _{v \in Q \cap E_{n}} \sigma\left(v-x_{n}, A\left(x_{n}\right)\right) \geqslant-\frac{1}{n},
$$

и, следовательно,

$$
\left\langle x_{n}, x_{n}^{*}\right\rangle \leqslant\left\langle v, x_{n}^{*}\right\rangle+\frac{1}{n} \quad \forall v \in Q \cap E_{n} .
$$

Докажем, что $\mathfrak{M}_{n}=\varnothing$ при больших $n$. В предположении противного $\mathfrak{M}_{n} \neq \varnothing$ для бесконечного числа номеров $n$, поэтому, не ограничивая обшности, можно считать, что $\mathfrak{M}_{n} \neq \varnothing$ для всех $n$. В частности, при любом $n$ сушествуют $x_{n}$ из $\mathfrak{M}_{n}, x_{n}^{*}$ из $A\left(x_{n}\right)$, удовлетворяюшие неравенству (5). Последовательности $x_{n}, x_{n}^{*}$ ограничены в пространствах $X, X^{*}$. Так как $X$ рефлексивно, то эти последовательности можно считать слабо сходящимися к элементам $x, x^{*}$. Оценка (5) влечет неравенство $\left\langle v, x^{*}\right\rangle \geqslant \overline{\lim }_{n \rightarrow \infty}\left\langle x_{n}, x_{n}^{*}\right\rangle$. Поскольку $x \in Q$, а последовательность $E_{n}$ исчерпьвает $Q$, то из последнего неравенства вытекает оценка

$$
\varlimsup_{n \rightarrow \infty}\left\langle x_{n}, x_{n}^{*}\right\rangle \leqslant\left\langle x, x^{*}\right\rangle
$$

Таким образом, последовательности $x_{n}, x_{n}^{*}$ обладают свойствами (1), поэтому имеют место соотношения (2), в частности $x \in \mathfrak{M}$. Используя (5), получаем $\left\langle x, x^{*}\right\rangle \leqslant\left\langle v, x^{*}\right\rangle(v \in Q)$, следовательно, $0 \in A(x)+N_{Q}(x)$. Это противоречит невырожденности оператора $A$ на $\mathfrak{M}$.

Считая $\mathfrak{M}_{k}=\varnothing$, положим $M=\left\{x \in Q, d_{X}(x, \mathfrak{M}) \leqslant 1 / k\right\}, E=E_{k}, \delta=1 /(2 k)$. Тогда

$$
\min _{v \in Q \cap E} \sigma(v-x, A(x))<-\delta \quad \forall x \in M .
$$

Фиксируем $y$ из $M$. Подберем вектор $v(y)$ из $\operatorname{ri}_{E}(Q \cap E)$ так, что $\sigma(v(y)-y$, $A(y))<-\delta$. Для каждой точки $y$ существует ее окрестность $O_{y}$ такая, что

$$
\sigma(v(y)-z, A(z)) \leqslant \sigma(v(y)-y, A(z))+\sigma(y-z, A(z))<-\delta \quad \forall z \in O_{y} .
$$

Впишем в покрытие $O_{y}(y \in M)$ метрического пространства $M$ локально конечное покрытие $V_{j}(j \in J)$. Пусть $\varphi_{j}(j \in J)$ - разбиение единицы класса $\Lambda(M)$, подчиненное покрытию $V_{j}$. Векторное поле

$$
w_{0}(x)=\sum_{j \in J} \varphi_{j}(x) v\left(y_{j}\right)
$$


(элемент $y_{j}$ подобран так, что $V_{j} \subset O_{y_{j}}$ ) обладает свойствами: $w_{0}(x) \in \operatorname{ri}_{E}(Q \cap E)$, $\sigma\left(w_{0}(x)-x, A(x)\right)<-\delta \forall x \in M$.

Пусть $\psi(t)=1$ при $t \leqslant 1, \psi(t)=2-t(1<t<2)$ и $\psi(t)=0$ при $t \geqslant 2$. Фиксируем элемент $w_{1}$ из $\operatorname{ri}_{E}(Q \cap E)$. Искомое векторное поле $w: Q \rightarrow X$ определим равенством

$$
w(x)=\psi\left(3 k d_{X}(x, \mathfrak{M})\right) w_{0}(x)+\left(1-\psi\left(3 k d_{X}(x, \mathfrak{M})\right)\right) w_{1}
$$

Поскольку $\psi\left(3 k d_{X}(x, \mathfrak{M})\right)=0$ для $x$ из $Q \backslash M$, способ продолжения $w_{0}$ на $Q \backslash M$ несуществен. Включение $w \in \Lambda(Q, X)$ очевидно; для проверки (4) достаточно учесть соотношения: $w(x)=w_{1}(x \in Q \backslash M), w(x)=w_{0}(x)(x \in \mathfrak{M})$. Лемма доказана.

Пусть $\Omega$ - ограниченное открытое (в относительной топологии) подмножество $Q, \partial_{Q} \Omega$ - относительная граница множества $\Omega$ и $\partial_{Q} \Omega \neq \varnothing$. Если оператор класса $S(Q)$ невырожден на $\partial_{Q} \Omega$, то согласно лемме 1 существуют пространство $E$ класса $\Gamma(Q)$ и векторное поле $w$ класса $\Lambda(Q, E)$ такие, что

$$
w(x) \in \operatorname{ri}_{E}(Q \cap E), \quad x \in Q, \quad\left\langle x-w(x), x^{*}\right\rangle>0, \quad x \in \partial_{Q} \Omega, \quad x^{*} \in A(x) .
$$

В частности, поле $I-w$ невырождено на $\partial_{Q} \Omega$ и определено относительное вращение $\gamma\left(I-w, \partial_{Q} \Omega\right)$ поля $I-w$ на $\partial_{Q} \Omega[4]$, [5]. Это вращение не зависит от произвола в способе выбора пространства $E$ и поля $w$, подчиненных требованиям (6). Число $\gamma\left(I-w, \partial_{Q} \Omega\right)$ назовем относительным вращением поля $A$ на $\partial_{Q} \Omega$ и обозначим символом $\gamma_{Q}(A, \Omega)$. Если $Q \in \mathscr{B}(X)$, то нуждается в особом рассмотрении случай $\Omega=Q$. В данной ситуации относительная граница $\partial_{Q} \Omega$ есть пустое множество. Следуя и в этом случае [4], [5], положим $\gamma_{Q}(A, Q)=\gamma(I-w, \varnothing)=1$.

Несколько иные, но приводящие к тем же результатам способы определения $\gamma_{Q}(A, \Omega)$ содержатся в [14], [15]. Относительное врашение гомотопически инвариантно и аддитивно. Обсуждение этих свойств можно найти в [15]; здесь же приведем лишь один результат.

Два оператора $A_{0}, A_{1}$ класса $S(Q)$ назовем сонаправленныци на множестве $\mathfrak{M} \subset Q$, если $0 \bar{\epsilon}(1-\lambda) A_{0}(x)+\lambda A_{1}(x) \quad(x \in \mathfrak{M}, 0 \leqslant \lambda \leqslant 1)$. Сонаправленность операторов $A_{0}, A_{1}$ на $\mathfrak{M}$ обеспечивает линейную гомотопность на $\mathfrak{M}$ соответствующих векторных полей. Из гомотопической инвариантности вращения следует

ПРЕДЛОЖЕНИЕ 1. Пусть операторы $A_{0}, A_{1}$ класса $S(Q)$ сонаправлены на границе $\partial_{Q} \Omega$ ограниченного открытого множества $\Omega \subset Q$. Тогда $\gamma_{Q}\left(A_{0}, \Omega\right)=\gamma_{Q}\left(A_{1}, \Omega\right)$.

Множество $\mathfrak{N} \subset Q$ называют особым для поля $A$ класса $S(Q)$, если любой элемент $u$ из $\mathfrak{N}$ есть особая точка оператора $A$. Особое множество изолировано, если в некоторой его относительной окрестности нет других особых точек поля $A$. Пересечение множества особых точек поля $A$ класса $S(Q)$ с любым шаром $\{x \in X,\|x\| \leqslant R\}$ компактно [14]. Если $\mathfrak{N}$ - ограниченное изолированное особое множество поля $A$ из $S(Q)$, то для ограниченных относительных окрестностей $O$ 
множества $\mathfrak{N}$ таких, что поле $A$ невырождено на $O \backslash \mathfrak{N}$, относительное вращение $\gamma_{Q}(A, O)$ одинаково; оно назьвается индексом особого множества $\mathfrak{N}$ поля $A$ и обозначается символом $\operatorname{ind}_{Q}(\mathfrak{N}, A)$.

Пусть $Y$ - замкнутое подпространство пространства $X, i_{Y}: Y \rightarrow X$-оператор вложения, $i_{Y}^{*}: X^{*} \rightarrow Y^{*}$ - сопряженньй к нему оператор. Если $Q \cap Y \neq \varnothing$, то каждому оператору $A: Q \rightarrow X^{*}$ можно сопоставить его след на $Q \cap Y$, обозначаемый символом $A_{Y}$ и определяемый равенством $A_{Y}=j_{Y}^{*} A j_{Y}$. Как нетрудно убедиться, след оператора $A$ класса $S(Q)$ на пространство $Y$ есть оператор класса $S(Q \cap Y)$.

Операторы класса $S(Q)$ оказываются полезными при изучении нелинейных функционалов. Пусть $f \in \Lambda(Q), x \in Q, v \in X_{0}=\operatorname{Lin}(Q-Q)$. Множество последовательностей $\{y, t\}$ таких, что $y \rightarrow x, t \rightarrow+0,[y, y+t v] \subset Q$, непусто [20]. Положим

$$
f^{0}(x, v)=\lim _{y \rightarrow x, t \rightarrow+0,[y, y+t v] \subset Q} \frac{f(y+t v)-f(y)}{t} .
$$

Число $f^{0}(x, v)$ называют обобщенной производной функционала $f$ в точке $x$ по направлению $v$. Функционал $f^{0}(x, \cdot): X_{0} \rightarrow \mathbb{R}$ выпукл, положительно-однороден, удовлетворяет условию Липшица и допускает однозначное продолжение с сохранением указанных свойств на пространство $X=\bar{X}_{0}$. За продолженным функционалом сохраним то же обозначение. Функционал $f^{0}(x, \cdot): X_{0} \rightarrow \mathbb{R}$ есть опорная функция множества класса $\mathrm{Cv}\left(X^{*}\right)$, обозначаемого символом $\partial f(x)$ и называемого обобщенным градиентом функиионала $f$ в точке $x$ [7], [20].

Через $\Lambda_{1}(Q)$ обозначим часть $\Lambda(Q)$, состояшую из функционалов $f$, для которых градиентное отображение $A(x)=\partial f(x), \quad x \in Q$, принадлежит классу $S(Q)$. В этом случае функционал $f$ назовем потенииалом оператора $A$, особые точки оператора $A$ - критическими точками функиионала $f$, неособые точки $A$ - регулярными точками $f$. Каждая точка локального минимума функционала $f$ является критической, поэтому любая окрестность регулярной точки $x$ содержит точку $y$, для которой $f(y)<f(x)$. Если поверхность уровня $f^{-1}(c)=$ $\{x \in Q, f(x)=c\}$ функционала $f$ пересекается с множеством $\mathfrak{C}_{f}$ его критических точек, то число $c$ именуют критическим значением функиионала $f$; в противном случае $\mathfrak{C}_{f} \cap f^{-1}(c)=\varnothing$ и $c$ - регулярное значение $f$.

Функционалы класса $\Lambda_{1}(Q)$ слабо полунепрерывны снизу [14]. Если $f \in \Lambda_{1}(Q)$ и при некотором $t<\infty$ его нижнее лебегово множество $M_{t}=\{x \in Q, f(x) \leqslant t\}$ непусто и ограничено, то $f$ достигает своего минимума на $Q$, множество $\arg \min f$ точек минимума $f$ принадлежит $\mathscr{K}(Q)$.

Пусть $Y$ - замкнутое подпространство пространства $X$ и $Q \cap Y \neq \varnothing$. Сужение функционала $f$ класса $\Lambda(Q)$ на $Q \cap Y$ есть функционал класса $\Lambda(Q \cap Y)$, обозначаемый символом $f_{Y}$. Справедливы равенства $\partial f_{Y}=j_{Y}^{*}(\partial f) j_{Y}=(\partial f)_{Y} ;$ в частности, если $f \in \Lambda_{1}(Q)$, то $f_{Y} \in \Lambda_{1}(Q \cap Y)$. В случае конечномерного пространства $Y$ функционал $f_{Y}$ называют конечномерной аппроксимацией $f$.

2. При определенных предположениях топологические характеристики функционала и его подходящих аппроксимаций совпадают. Для формулировки и доказательства соответствуюших результатов потребуются некоторые геометрические понятия и факты. Пусть $\mathscr{H}$ - конечномерное евклидово пространство с нор- 
мой $|\cdot|, \quad B=\{x \in \mathscr{H},|x|<1\}$ - открытый шар радиуса 1 с центром в нуле. Элемент $v$ из $\mathscr{H} \backslash\{0\}$ называют $[7$, с. 59] гиперкасательной $к$ множеству $\mathscr{D} \subset \mathscr{H}$ в точке $x \in \mathscr{D}$, если сушествует такое $\varepsilon>0$, что

$$
y+t w \in \mathscr{D}, \quad y \in(x+\varepsilon B) \cap \mathscr{D}, \quad w \in v+\varepsilon B, \quad 0<t<\varepsilon .
$$

Из существования одной гиперкасательной к множеству $\mathscr{D}$ в точке $x$ следует, что множество $\mathrm{T}_{\mathscr{D}}(x)$ всех таких гиперкасательных есть непустой открытый выпукльй конус в $\mathscr{H}\left[7\right.$, c. 59]. Очевидно, что Т $\mathscr{D}(x)=\mathscr{H}$ для $x$ из $\mathscr{D}^{0}$, поэтому интересен случай, когда $x$ - граничная точка множества $\mathscr{D}$. Различные способы описания конуса $\mathrm{T}_{\mathscr{D}}(x)$ рассмотрены в $[7$, гл. 2]. Если, например,

$$
\mathscr{D}=\{y \in \mathscr{H}, g(y) \leqslant 0\}, \quad g \in \Lambda(\mathscr{H}), \quad g(x)=0, \quad 0 \bar{\epsilon} \partial g(x),
$$

то $\left\{v \in \mathscr{H}, g^{0}(x, v)<0\right\} \subset \mathrm{T}_{\mathscr{D}}(x)$; при некоторых дополнительных ограничениях на функцию $g$ справедливо равенство $\left\{v \in \mathscr{H}, g^{0}(x, v)<0\right\}=\mathrm{T}_{\mathscr{D}}(x)$. Если $\mathscr{D} \in \operatorname{Cv}(\mathscr{H})$ и $\mathscr{D}^{0} \neq \varnothing$, то $\mathrm{T}_{\mathscr{D}}(x)=T_{\mathscr{D}}^{0}(x), \quad x \in \mathscr{D}$.

Множество $\mathscr{D}$ класса $\mathscr{K}(\mathscr{H})$ назовем сильно липшицевым, если $\mathrm{T}_{\mathscr{D}}(x) \neq \varnothing$ $\forall x \in \mathscr{D}$. Требование $\mathrm{T}_{\mathscr{D}}(x) \neq \varnothing$ обеспечивает равенство $\mathscr{D}=\overline{\mathscr{D}}^{0}$. Для сильно липшицева множества $\mathscr{D}$ конечны все числа Бетти $b_{l}(\mathscr{D})$ и $b_{l}(\mathscr{D})=0$ при $l>\operatorname{dim} \mathscr{H}[21],[22]$. Поэтому имеет смысл эйлерова характеристика $\mathrm{X}(\mathscr{D})$ множества $\mathscr{D}$.

Лемма 2. Пусть множество $\mathscr{D} \subset \mathscr{H}$ сильно липшицево, $v \in \Lambda(\mathscr{D}, \mathscr{H})$ u $v(x) \in \mathrm{T}_{\mathscr{D}}(x), \quad x \in \mathscr{D}$. Тогда:

1) при любом z из $\mathscr{D}$ задача Коши

$$
\frac{d x}{d t}=v(x), \quad x(0)=z
$$

имеет единственное решение $x(t ; z), t \geqslant 0, \quad z \in \mathscr{D}$;

2) определяемое равенством $U_{t} z=x(t ; z)$ семейство операторов сдвига $U_{t}: \mathscr{D} \rightarrow \mathscr{D}, t \geqslant 0$, образует непрерывную полугруппу;

3) вращение $\gamma\left(-v, \mathscr{D}^{0}\right)$ поля $-v$ на границе $\partial \mathscr{D}$ множества $\mathscr{D}$ совпадает $с$ эйлеровой характеристикой множества $\mathscr{D}: \gamma\left(-v, \mathscr{D}^{0}\right)=\mathrm{X}(\mathscr{D})$.

ДокАЗАТЕЛЬСТво. Однозначная разрешимость задачи (7) для $z$ из $\mathscr{D}^{0}$ вытекает из классических результатов теории дифференциальных уравнений. Соответствуюшая траектория $x(t ; z)$ не пересекается с $\partial \mathscr{D}$, это стандартным образом выводится из включения $v(x) \in \mathrm{T}_{\mathscr{D}}(x)$, означающего, что поле $v$ направлено внутрь множества $\mathscr{D}$. Отображение $(z, t) \rightarrow x(t ; z)$, заданное первоначально на $\mathscr{D}^{0} \times \mathbb{R}_{+}$, допускает продолжение по непрерывности на $\mathscr{D} \times \mathbb{R}_{+} \cdot$ В частности, операторы $U_{t}=x(t ; \cdot): \mathscr{D} \rightarrow \mathscr{D}$ образуют семейство операторов, непрерывно зависяших от параметра $t \geqslant 0$. Из определения операторов $U_{t}$ вытекают равенства $U_{0}=I$, $U_{t_{1}+t_{2}}=U_{t_{1}} U_{t_{2}}, t_{1} \geqslant 0, t_{2} \geqslant 0$, означаюшие, что семейство $U_{t}: \mathscr{D} \rightarrow \mathscr{D}, t \geqslant 0$, образует полугруппу. 
Условие $v(x) \in \mathrm{T}_{\mathscr{D}}(x)$ обеспечивает включение $U_{t} \mathscr{D} \subset \mathscr{D}^{0}$ при $t>0$. Поэтому векторные поля $I-U_{t}, t>0$, невырождены на $\partial \mathscr{D}$. Как известно [6, с. 71], $\gamma\left(I-U_{t}, \mathscr{D}^{0}\right)=\gamma\left(-v, \mathscr{D}^{0}\right), t>0$. С другой стороны, согласно формуле Лефшеца-Хопфа [21], [22, с. 261] $\gamma\left(I-U_{t}, \mathscr{D}^{0}\right)=\operatorname{Le}\left(U_{t}\right)$, где $\operatorname{Le}\left(U_{t}\right)$ - число Лефшеца отображения $U_{t}: \mathscr{D} \rightarrow \mathscr{D}$, совпадаюшего ввиду гомотопности отображений $I$, $U_{t}: \mathscr{D} \rightarrow \mathscr{D}$ с эйлеровой характеристикой множества $\mathscr{D}$. Лемма доказана.

Пусть $C \in \operatorname{Cv}(\mathscr{H}), C^{0} \neq \varnothing, \mathscr{D} \in \mathscr{K}(\mathscr{H}), \mathscr{D} \subset C$. Скажем, что отображение $\mathscr{F}: \partial_{C} \mathscr{D} \rightarrow \operatorname{Cv}(\mathscr{H})$ удовлетворяет условию $(N)$, если из соотношений $x \in \partial_{C} \mathscr{D}$, $u \in T_{C}^{0}(x), \sigma(u, \mathscr{F}(x))<0$ вытекает включение $u \in \mathrm{T}_{\mathscr{D}}(x)$.

Для проверки условий леммы 2 в указанных предположениях полезна

Лемma 3. Пусть $v \in \Lambda(\mathscr{D}, \mathscr{H}) u$

$$
v(x) \in T_{C}^{0}(x) \quad \forall x \in \mathscr{D}, \quad \sigma(v(x), \mathscr{F}(x))<0 \quad \forall x \in \partial_{C} \mathscr{D},
$$

где $\mathscr{F}: \partial_{C} \mathscr{D} \rightarrow \operatorname{Cv}(\mathscr{H})$ - отображение, удовлетворяющее условию $(N)$. Тогда множество $\mathscr{D}$ сильно липшицево и $v(x) \in \mathrm{T}_{\mathscr{D}}(x), \quad x \in \mathscr{D}$.

ДокАЗАТЕЛЬСТво. Если $x \in \partial_{C} \mathscr{D}$, то из условий $v(x) \in T_{C}^{0}(x)$, $\sigma(v(x), \mathscr{F}(x))<0$ и условия $(N)$ вытекает включение $v(x) \in \mathrm{T}_{\mathscr{D}}(x)$. Для $x$ из $\partial \mathscr{D} \backslash \partial_{C} \mathscr{D}$ имеем $\mathrm{T}_{\mathscr{D}}(x)=T_{C}^{0}(x)$, следовательно, $v(x) \in T_{C}^{0}(x)=\mathrm{T}_{\mathscr{D}}(x)$.

Итак, $v(x) \in \mathrm{T}_{\mathscr{D}}(x)$ для любого $x$ из $\partial \mathscr{D}$. Отсюда следует требуемое утверждение. Лемма доказана.

Введем бесконечномерный вариант условия $(N)$. Пусть $\Omega$ - ограниченное открытое (в относительной топологии) подмножество $Q$. Оператор $A$ класса $S(Q)$ назовем конормальным области $\Omega$, если $A$ невырожден на $\partial_{Q} \Omega$ и для любого пространства $E$ класса $\Gamma(Q)$ ( $E$ наделено евклидовой структурой) оператор $F=$ $A_{E}=j_{E}^{*} A j_{E}$ и множества $C=Q \cap E, \mathscr{D}=\bar{\Omega} \cap E$ удовлетворяют условию $(N)$. Так как $\partial_{Q \cap E}(\bar{\Omega} \cap E) \subset\left(\partial_{Q} \Omega\right) \cap E \subset \partial_{Q} \Omega$, то условие конормальности относится лишш к точкам из $\partial_{Q} \Omega$.

В качестве примера рассмотрим случай, когда $A=\partial f, f \in \Lambda_{1}(Q), \Omega=\{x \in Q$, $f(x)<c\}, c$-регулярное значение функционала $f$. Если $x \in \partial_{Q} \Omega$, то $f(x)=c$. Так как $c$ - регулярное значение $f$, то $x$ - неособая точка оператора $\partial f$. Следовательно, оператор $\partial f$ невырожден на $\partial_{Q} \Omega$. Пусть $E \in \Gamma(Q)$, пространство $E$ наделено евклидовой структурой, $x \in \partial_{Q \cap E}(\bar{\Omega} \cap E), u \in T_{Q \cap E}^{0}(x), f^{0}(x, u)<0$. Тогда $x \in\left(\partial_{Q} \Omega\right) \cap E, f(x)=c$. Из соотношений $f(x)=c, u \in T_{Q \cap E}^{0}(x), f^{0}(x, u)<0$ и результатов $[7$, гл. 2$]$ вытекает, что $u \in \mathrm{T}_{\bar{\Omega} \cap E}(x)$. Таким образом, оператор $\partial f$ конормален области $\Omega=\{x \in Q, f(x)<c\}$.

Свойство конормальности сохраняется при переходе к следам операторов. Если оператор $A$ класса $S(Q)$ конормален области $\Omega$, то его след $A_{Y}: Y \rightarrow Y^{*}$ на пространство $Y$ класса $\Gamma(Q)$ будет конормален области $\Omega$. Это непосредственно вытекает из определения конормальности. Значение понятия конормальности выясняет 
ТЕОРема 1. Пусть $\Omega$ - ограниченное открытое (в относительной топологии) подмножество множества $Q$. Пусть оператор А класса $S(Q)$ конормален области $\Omega$. Тогда:

1) существует такое пространство $Е$ из $\Gamma(Q)$, что если $Y \subset \Gamma(X)$, $E \subset Y$, то группы гомологий пространств $\bar{\Omega}$ и $\bar{\Omega} \cap Y$ изоморфны: $H_{l}(\bar{\Omega}) \approx$ $H_{l}(\bar{\Omega} \cap Y), l=0,1, \ldots$;

2) $\gamma_{Q}(A, \Omega)=\mathrm{X}(\bar{\Omega})$.

ДокаЗАТЕЛЬСтво. В вырожденном случае $\left(\Omega=Q \in \mathscr{B}(X), \partial_{Q} \Omega=\varnothing\right)$ утверждения 1), 2) очевидны, поэтому можно считать, что $\partial_{Q} \Omega \neq \varnothing$. Доказательство удобно разбить на отдельные этапы. Первый этап: построение полугрупшы операторов $U_{t}: \bar{\Omega} \rightarrow \bar{\Omega}$. Так как оператор $A$ класса $S(Q)$ невырожден на $\partial_{Q} \Omega$, то согласно лемме 1 сушествуют векторное поле $w$ из $\Lambda(Q, X)$, пространство $E$ класса $\Gamma(Q)$ и число $\delta>0$ такие, что имеют место соотношения (4) с $\mathfrak{M}=\partial_{Q} \Omega$.

Рассмотрим задачу Коши

$$
\frac{d x}{d t}=w(x)-x, \quad x(0)=z .
$$

Фиксируем $z$ из $\bar{\Omega}$ и положим $\mathscr{H}=\operatorname{Lin}(E, z), \mathscr{D}=\bar{\Omega} \cap \mathscr{H}, C=Q \cap \mathscr{H}$, $\mathscr{F}=A_{\mathscr{H}}=j_{\mathscr{H}}^{*} A j_{\mathscr{H}}, v(x)=w(x)-x$. Как отмечалось выше, $\mathscr{H} \in \Gamma(Q)$, поэтому $C^{0} \neq \varnothing$; считаем, что $\mathscr{H}$ наделено структурой евклидова пространства, операторы $j_{\mathscr{H}}, j_{\mathscr{H}}^{*}$ согласованы с этой структурой. Так как $w(x) \in \operatorname{ri}_{E}(Q \cap E)$, то $v(x)=w(x)-x \in T_{C}^{0}(x), x \in \mathscr{D}$. Поскольку оператор $A$ конормален области $\Omega$, то отображение $\mathscr{F}: \partial_{C} \mathscr{D} \rightarrow \operatorname{Cv}(\mathscr{H})$ удовлетворяет условию $(N)$. Если $x \in \partial_{C} \mathscr{D} \subset\left(\partial_{Q} \Omega\right) \cap \mathscr{H}$, то

$$
\sigma(v(x), \mathscr{F}(x))=\sigma(w(x)-x, A(x))<0 .
$$

Все условия леммы 3 выполнены, что согласно леммам 2,3 влечет существование решения $x(t ; z), t \geqslant 0$, задачи Коши (8), не выходяшего за пределы множества $\mathscr{D}: x(t ; z) \in \bar{\Omega} \cap \operatorname{Lin}(E, z), t \geqslant 0, z \in \bar{\Omega}$.

Включение $v \in \Lambda(Q, X)$ влечет непрерывность $x(t ; z)$ по совокупности переменных. Равенство $U_{t} z=x(t ; z), t \geqslant 0, z \in \bar{\Omega}$, определяет непрерывную полугруппу преобразований $U_{t}, t \geqslant 0$, оставляющую инвариантным не только множество $\bar{\Omega}$, но и его конечномерные сечения вида $\bar{\Omega} \cap Y, Y \supset E$.

Второй этап - аттрактор полугрупшы $U_{t}: \bar{\Omega} \rightarrow \bar{\Omega}$. Положим $\mathfrak{U}=$ $\bigcap_{t \geqslant 0} U_{t}(\bar{\Omega} \cap E)$. Множество $\mathfrak{U}$ есть непустой компакт в пространстве $E$; справедливы соотношения

$$
\lim _{t \rightarrow \infty} \theta_{X}\left(U_{t}(\bar{\Omega} \cap E), \mathfrak{U}\right)=0, \quad U_{t} \mathfrak{U}=\mathfrak{U} \quad \forall t \geqslant 0,
$$

выражающие свойство притяжения и свойство инвариантности множества $\mathfrak{U}$. Следовательно, $\mathfrak{U}$ есть минимальный глобальный аттрактор сужения полугруппы $U_{t}$ 
на множество $\bar{\Omega} \cap E[23]$. Из (8) и включения $w(x) \in E, x \in \bar{\Omega}$, легко выводится, что $\theta_{X}\left(U_{t}(\bar{\Omega}), E\right) \rightarrow 0$ при $t \rightarrow \infty$. Это позволяет гарантировать свойство притяжения в усиленной форме:

$$
\lim _{t \rightarrow \infty} \theta_{X}\left(U_{t}(\bar{\Omega}), \mathfrak{U}\right)=0 .
$$

Следовательно, множество $\mathfrak{U}$ класса $\mathscr{K}(E)$ есть аттрактор полугрупшы $U_{t}: \bar{\Omega} \rightarrow \bar{\Omega}$.

Третий этап - доказательство изоморфности груп $H_{l}(\bar{\Omega})$ и $H_{l}(\bar{\Omega} \cap E)$. Будем рассматривать $\mathscr{K}(\bar{\Omega})$ как направленное множество $[17$, с. 338$]$ относительно порядка, порождаемого включением. Обозначим через $\mathscr{K}_{1}(\bar{\Omega})$ часть $\mathscr{K}(\bar{\Omega})$, состоящую из компактов $\mathfrak{K}$, удовлетворяюших условиям:

1) $\mathfrak{U} \subset \mathfrak{K}$;

2) $U_{t}(\mathfrak{K}) \subset \mathfrak{K}, t \geqslant 0$.

Если $\mathfrak{K}_{0} \in \mathscr{K}(\bar{\Omega})$, то, используя (9), несложно показать, что содержащее $\mathfrak{K}_{0}$ множество $\mathfrak{K}=\bigcup_{t \geqslant 0} U_{t}\left(\mathfrak{K}_{0} \cup \mathfrak{U}\right)$ принадлежит $\mathscr{K}_{1}(\bar{\Omega})$. Таким образом, $\mathscr{K}_{1}(\bar{\Omega})$ есть конфинальная часть $\mathscr{K}(\bar{\Omega})$.

Пусть $P \in \mathscr{K}_{1}(\bar{\Omega}), P_{n}=U_{n}(P), n=0,1, \ldots, \Pi_{n}^{m}: P_{m} \rightarrow P_{n}, m \geqslant n,-$ отображения вложения. Пространство $\mathfrak{U}$ является пересечением спектра пространств $\left\{P_{n}, \Pi_{n}^{m}\right\}\left[18\right.$, c. 321]. При любых $m \geqslant n$ отображение вложения $\Pi_{n}^{m}: P_{m} \rightarrow P_{n}$ гомотопно гомеоморфизму $U_{n} U_{m}^{-1}: P_{m} \rightarrow P_{n}$. Соединяюшее $\Pi_{n}^{m}$ и $U_{n} U_{m}^{-1}$ семейство отображений $\Phi_{\lambda}: P_{m} \rightarrow P_{n}, 0 \leqslant \lambda \leqslant 1$, можно определить равенством $\Phi_{\lambda}=U_{(1-\lambda) m+\lambda n} U_{m}^{-1}$.

Фиксируем целое число $l \geqslant 0$. Индуцированный $\Pi_{n}^{m}$ гомоморфизм $\Pi_{n *}^{m}$ групп гомологий $H_{l}\left(P_{m}\right)$ и $H_{l}\left(P_{n}\right)$ является изоморфизмом. В силу свойства непрерьвности теории спектральных гомологий $[18$, с. 322$]$ индуцируемый вложением $i: \mathfrak{U} \rightarrow P$ гомоморфизм $i_{*}: H_{l}(\mathfrak{U}) \rightarrow H_{l}(P)$ также есть изоморфизм.

Пусть $P^{\prime} \in \mathscr{K}_{1}(\bar{\Omega}), P \subset P^{\prime}$ и $k=k\left(P, P^{\prime}\right): P \rightarrow P^{\prime}$ - оператор вложения. Для индуцируемого вложением $j=k i: \mathfrak{U} \rightarrow P^{\prime}$ гомоморфизма $j_{*}: H_{l}(\mathfrak{U}) \rightarrow H_{l}\left(P^{\prime}\right)$ верно равенство $j_{*}=k_{*} i_{*}$. Так как $j_{*}, i_{*}$ есть изоморфизмы, то и $k_{*}=$ $k_{*}\left(P, P^{\prime}\right): H_{l}(P) \rightarrow H_{l}\left(P^{\prime}\right)$ также есть изоморфизм. По определению гомологий с компактными носителями $\left[17\right.$, c. 244] $H_{l}(\bar{\Omega})$ есть предел прямого спектра групп $\left\{H_{l}(P), k_{*}\left(P, P^{\prime}\right)\right\}, \quad P \in \mathscr{K}_{1}(\bar{\Omega}), \quad P^{\prime} \in \mathscr{K}_{1}(\bar{\Omega})$. Отсюда вытекает изоморфизм групп $H_{l}(\mathfrak{U}), H_{l}(\bar{\Omega}), H_{l}(\bar{\Omega} \cap E)$. В предшествуюших рассуждениях можно всюду заменить пространство $X$ пространством $Y$ класса $\Gamma(Q), E \subset Y$. Это и доказывает изоморфизм $H_{l}(\bar{\Omega}) \approx H_{l}(\bar{\Omega} \cap Y), l=0,1, \ldots, Y \supset E$.

Четвертый этап - доказательство равенства $\gamma_{Q}(A, \Omega)=\mathrm{X}(\bar{\Omega}) . \quad$ Пусть $\mathscr{D}=\bar{\Omega} \cap E$. Так как $H_{l}(\bar{\Omega}) \approx H_{l}(\mathscr{D}), \quad l=0,1, \ldots$, то определены и совпадают эйлеровы характеристики множеств $\bar{\Omega}$ и $\mathscr{D}: \mathrm{X}(\bar{\Omega})=\mathrm{X}(\mathscr{D})$. Согласно лемме 2 $\mathrm{X}(\mathscr{D})=\gamma\left(I-w, \mathscr{D}^{0}\right)$. Используя включение $w(x) \in \operatorname{ri}_{E}(Q \cap E)$ и известные свойства относительного вращения [4], [5], приходим к равенству

$$
\gamma\left(I-w, \mathscr{D}^{0}\right)=\gamma\left(I-w, \partial_{Q} \Omega\right) .
$$

Но $\gamma\left(I-w, \partial_{Q} \Omega\right)=\gamma_{Q}(A, \Omega)$ в силу определения $\gamma_{Q}(A, \Omega)$ (см. п. 1$)$. Итак, имеют место равенства

$$
\mathrm{X}(\bar{\Omega})=\mathrm{X}(\mathscr{D})=\gamma\left(I-w, \mathscr{D}^{0}\right)=\gamma\left(I-w, \partial_{Q} \Omega\right)=\gamma_{Q}(A, \Omega) .
$$


Теорема доказана.

СЛЕДСТВИЕ 1. Пусть $f \in \Lambda_{1}(Q), a-$ - регулярное значение функционала $f$, лебегово множество $M_{a}=\{x \in Q, f(x) \leqslant a\}$ непусто и ограничено. Тогда:

1) найдется такое пространство $E$ класса $\Gamma(Q)$, что если $Y \in \Gamma(X)$ и $E \subset Y, \operatorname{mo~}_{l}\left(M_{a}\right) \approx H_{l}\left(M_{a} \cap Y\right), l=0,1, \ldots$;

2) $\gamma\left(\partial f, M_{a}^{0}\right)=\mathrm{X}\left(M_{a}\right)$.

Для доказательства достаточно положить $A=\partial f, \Omega=\{x \in Q, f(x)<a\}$ и воспользоваться теоремой 1. Следствие означает совпадение топологических характеристик у функционала $f$ и некоторых его конечномерных аппроксимаций. Комбинация теоремы 1 с предложением 1 влечет

СЛЕДСТВИЕ 2. Пусть операторы $A_{0}, A_{1}$ класса $S(Q)$ сонаправлены на граниче ограниченного открытого множества $\Omega \subset Q$ и один из них конормален области $\Omega$. Тогда $\gamma_{Q}\left(A_{0}, \Omega\right)=\gamma_{Q}\left(A_{1}, \Omega\right)=\mathrm{X}(\bar{\Omega})$.

Теорема 1 представляет бесконечномерный многозначный вариант теоремы Пуанкаре-Хопфа (см., например, [8, с. 172]). Ее относительный вариант $(Q \neq X)$ не был известен и для случая $\operatorname{dim} X<\infty$. В бесконечномерном пространстве $X$ содержательным и новым является и первое утверждение теоремы.

3. Для широкого класса функционалов на банаховом пространстве (многообразии) верен следующий принцип: если отрезок $[a, b]$ не содержит критических значений функционала $f$, то множество $[f \leqslant a]$ является деформационным ретрактом множества $[f \leqslant b]$. В работе [12] установлен вариант этого принципа для липшицевых функционалов на банаховом многообразии. Его относительная версия для негладких функций конечного числа переменных доказана в $[11$, с.76]. Ниже результат из [11] переносится на бесконечномерный случай.

В пределах этого пункта $f \in \Lambda_{1}(Q), M_{\alpha}=\{x \in Q, f(x) \leqslant \alpha\}, M_{\alpha, \beta}=$ $\{x \in Q, \alpha \leqslant f(x) \leqslant \beta\}, \mathscr{G}_{\alpha, \beta}=\{x \in Q, \alpha<f(x)<\beta\},-\infty<\alpha<\beta<\infty$. Для изучения топологических характеристик функционала $f$ будут использоваться непрерывные деформации, при конструировании которых окажется полезной

ЛЕМма 4. Пусть поле $w$ из $\Lambda(Q, X)$ удовлетворяет условиям

$$
\begin{gathered}
w(x) \in \operatorname{ri}_{E}(Q \cap E), \quad x \in \mathscr{G}_{\alpha, \beta}, \\
f^{0}(x, w(x)-x)<-\delta, \quad x \in M_{\alpha, \beta},
\end{gathered}
$$

где $\delta>0, E \in \Gamma(Q)$. Тогда:

1) при любом z из $f^{-1}((\alpha, \beta])$ задача Коши (8) имеет единственное решение $x(t ; z), \quad 0 \leqslant t \leqslant \tau(z)$, функиия $g_{z}(t)=f(x(t ; z))$ убивает на $[0, \tau(z)]$ и $g_{z}(\tau(z))=\alpha$;

2) функиии $\tau(z)$ и $x(t ; z)$ могут быть продолжены по непрерывности на множества $M_{\alpha, \beta} u\left\{(t, z), z \in M_{\alpha, \beta}, 0 \leqslant t \leqslant \tau(z)\right\}$ соответственно. 
ДокАЗАТЕЛЬСТво. Повторяя рассуждения, проведенные на первом этапе доказательства теоремы 1 , можно установить сушествование решения задачи (8) для $z$ из $\mathscr{G}_{\alpha, \beta}$. Поскольку $f^{-1}(\beta) \subset \overline{\mathscr{G}}_{\alpha, \beta}, w \in \Lambda(Q, X)$, то решение $x(t ; z)$ задачи (8) существует и единственно для всех $z$ из $f^{-1}((\alpha, \beta])$. В силу $(11),(8)$ верхняя правая производная $g_{z}^{\prime}(t)$ функции $g_{z}(t)=f(x(t ; z))$ удовлетворяет оценке $g_{z}^{\prime}(t)<-\delta$ на том промежутке $[0, \tau]$, где $g_{z}(t) \geqslant \alpha$. В частности, время $\tau(z)$ перехода точки из начального положения $z$ на поверхность $f^{-1}(\alpha)$ по траектории уравнений (8) удовлетворяет оценке $0<\tau(z)<(f(z)-\alpha) / \delta$. Положим $\tau(z)=0$, $x(0 ; z)=z$, если $f(z)=\alpha$.

Убедимся в том, что определенные таким образом функции $\tau(z), x(t ; z)$ являются искомыми. Непрерьвность функции $\tau(\cdot)$ на множестве $f^{-1}((\alpha, \beta])$ вытекает из равенства $g_{z}(\tau(z))=\alpha$ и простейших теорем о неявных функциях (повторяя рассуждения, проведенные при доказательстве леммы 4 работы [22], можно показать, что функция $\tau(\cdot)$ удовлетворяет локальному условию Липшица). Оценка $0<\tau(z)<(f(z)-\alpha) / \delta, \alpha<f(z) \leqslant \beta$, влечет непрерывность функции $\tau(\cdot)$ и в точках поверхности $f^{-1}(\alpha)$. Непрерывность функции $x(t ; z)$ на соответствующем множестве следует из включения $v \in \Lambda(Q, X)$. Лемма доказана.

Теорема 2. Пусть отрезок $[a, b]$ не содержит критических значений функиионала $f$ класса $\Lambda_{1}(Q)$ и множество $M_{a, b}$ ограничено. Тогда множество $M_{a}$ есть деформационный ретракт множества $M_{b}$.

ДоказАтельство. Так как оператор $A=\partial f$ невырожден на ограниченном замкнутом множестве $\mathfrak{M}=M_{a, b}$, то в силу леммы 1 сушествуют $E$ из $\Gamma(Q)$, отображение $w$ класса $\Lambda(Q, E)$ и постоянная $\delta>0$ такие, что выполнены соотношения (4). Отсюда следует, что выполнены условия (10), (11) при $\alpha=a, \beta=b$. Согласно лемме 4 сушествуют функции $\tau(z), x(t ; z)$, обладаюшие указанными в ней свойствами 1$), 2)$. Определим на $M_{b} \times[0,1]$ отображение $V$ равенством

$$
V(z, \lambda)= \begin{cases}x(\lambda \tau(z) ; z), & \text { если } z \in M_{a, b} \\ z, & \text { если } z \in M_{a}\end{cases}
$$

Отображение $V: M_{b} \times[0,1] \rightarrow M_{b}$ непрерывно и справедливы соотношения:

$$
\begin{aligned}
& V(z, 0)=z \quad \forall z \in M_{b}, \\
& V(z, \lambda)=z, \quad z \in M_{a}, \quad 0 \leqslant \lambda \leqslant 1, \\
& V(z, 1) \in M_{a} \quad \forall z \in M_{b} .
\end{aligned}
$$

Эти соотношения без труда выводятся из отмеченных выше свойств $\tau(z), x(t ; z)$. Они означают, что $M_{a}$ есть деформационньй ретракт $M_{b}$. Теорема доказана.

Из доказательства теоремы 2 видно, что отображение $V(\cdot, \lambda)$ оставляет инвариантным множество $M_{b} \cap E$, а пара $\left(M_{a}, M_{a} \cap E\right)$ является деформационным ретрактом пары $\left(M_{b}, M_{b} \cap E\right)$. Аналогичный результат для гладких функционалов с невырожденными критическими точками установлен в [16, с. 211]. 
В условиях теоремы 2 группы гомологий пространств $M_{a}, M_{b}$ изоморфны:

$$
H_{l}\left(M_{a}\right) \approx H_{l}\left(M_{b}\right), \quad l=0,1, \ldots
$$

Близкие, но иные, достаточные для справедливости соотношения (12), условия предлагает

ТЕОРема 3. Пусть лебегово множество $M_{b}$ функционала $f$ класса $\Lambda_{1}(Q)$ непусто $и$ ограничено, $a=\min \{f(x), x \in Q\}<b \quad u$ промежуток $(a, b]$ не содержит критических значений $f$. Тогда имеет место (12) и $\operatorname{ind}\left(M_{a}, \partial f\right)=\mathrm{X}\left(M_{a}\right)$.

ДокАЗАТЕльСтво. Для упрощения записи будем считать, что $a=0, b=1$. Пусть $c=1 / 2, c_{n}=1 / 2^{n+1}, d_{n}=3 c_{n}, n=1,2, \ldots$ Построим систему функций $\varphi_{n}$ класса $\Lambda(\mathbb{R})$, удовлетворяющих условиям:

1) $\varphi_{n}(t) \geqslant 0, \sum_{n=1}^{\infty} \varphi_{n}(t)=1,0<t \leqslant c$;

2) $\left\{t: \varphi_{n}(t)>0\right\}=\left(p_{n}, q_{n}\right), c_{n}<p_{n}<q_{n}<d_{n}, n=1,2, \ldots$

Система интервалов $\left(p_{n}, q_{n}\right), n=1,2, \ldots$, образует открытое покрытие промежутка $(0, c]$ кратности 2 .

Положим $\mathfrak{M}_{n}=\left\{x \in Q, c_{n} \leqslant f(x) \leqslant d_{n}\right\}$. Множество $\mathfrak{M}_{n}$ ограничено, замкнуто и не содержит особых точек поля $A=\partial f$. Согласно лемме 1 сушествуют $E_{n}$ из $\Gamma(Q)$, поле $w_{n}$ класса $\Lambda(Q, X)$ и постоянная $\delta_{n}>0$ такие, что

$$
w_{n}(x) \in \operatorname{ri}_{E_{n}}\left(Q \cap E_{n}\right) \quad \forall x \in Q, \quad f^{0}\left(x, w_{n}(x)-x\right)<-\delta_{n} \quad \forall x \in \mathfrak{M}_{n} .
$$

Рассмотрим поле

$$
w(x)=\sum_{n=1}^{\infty} \varphi_{n}(f(x)) w_{n}(x), \quad x \in Q .
$$

Очевидно, что $w \in \Lambda\left(Q \backslash M_{0}, X\right)$ и $w(x)=0$, если $f(x) \geqslant 3 / 4$. Из (13) вытекает оценка

$$
f^{0}(x, w(x)-x)<-h(f(x)), \quad x \in M_{c} \backslash M_{0},
$$

где $h:(0, c] \rightarrow \mathbb{R}$ - положительная возрастающая функция.

Пусть $(\alpha, \beta)$ - один из интервалов смежности открытого множества $\mathscr{G}=$ $\left\{t \in(0, c), t \neq p_{n}, t \neq q_{n}, n=1,2, \ldots\right\}$. Тогда на $(\alpha, \beta)$ все функции $\varphi_{n}$ (кроме одной из двух) равны нулю. Обозначим через $E$ линейную оболочку $\cup E_{i}$, где $i$ таковы, что $\varphi_{i}(t)>0$ на $(\alpha, \beta)$. Из (13) следует включение

$$
w(x) \in \operatorname{ri}_{E}(Q \cap E), \quad \text { если } \quad \alpha<f(x)<\beta .
$$

Векторное поле $v_{0}(x)$, совпадающее с $w(x)-x$ на $Q \backslash M_{0}$ и равное нулю на $M_{0}$, может не входить в класс $\Lambda(Q, X)$, однако для любого $z$ из $M_{c}$ задача Коши

$$
\frac{d x}{d t}=v_{0}(x), \quad x(0)=z
$$


имеет единственное решение $x(t ; z)$. Действительно, если $z \in M_{0}$, то $x(t ; z) \equiv z$ при $t \geqslant 0$. Для каждого $z$ из $M_{c} \backslash M_{0}$ найдется такой интервал смежности $(\alpha, \beta)$ множества $\mathscr{G}$, что $\alpha<f(z) \leqslant \beta$. Рассмотрим векторное поле

$$
v(x)=\psi\left(\frac{f(x)}{\varepsilon}\right) v_{0}(x)
$$

где $\alpha>\varepsilon>0, \psi(t)=0$ при $t \leqslant 0, \psi(t)=t$ при $0<t<1, \psi(t)=1$ при $t \geqslant 1$. Разрешимость задачи Коши $\frac{d x}{d t}=v(x), x(0)=z$ на промежутке $[0, \tau(z)]$ вытекает из $(14),(15)$ и леммы 4 , соответствуюшее решение не покидает множества $M_{\alpha, \beta}$, на котором поля $v, v_{0}$ одинаковы. Тем самым установлено сушествование решения $x(t ; z)$ задачи (16) на промежутке $[0, \tau(z)]$, при этом $f(x(\tau(z) ; z))=\alpha$. Полученное решение с помощью аналогичной конструкции может быть продолжено на всю полуось $t \geqslant 0$.

Фиксируем $z$ из $M_{c} \backslash M_{0}$ и рассмотрим функцию $g_{z}(t)=f(x(t ; z)), t \geqslant 0$. Ее верхняя правая производная удовлетворяет вытекающему из (14), (16) неравенству $\frac{d}{d t} g_{z}(t)<-h\left(g_{z}(t)\right)$, непосредственным следствием которого является равенстBO

$$
\lim _{t \rightarrow \infty} f(x(t ; z))=0
$$

равномерное относительно $z$ из множества $M_{c} \backslash M_{0}$. При любом $z$ из $M_{c} \backslash M_{0}$ функция $g_{z}(t)$ убывает. В частности, определяемое равенством $U_{t} z=x(t ; z)$ семейство операторов $U_{t}, t \geqslant 0$, оставляет инвариантными множества $M_{d}, 0 \leqslant d \leqslant c$.

Из результатов работы [14] вытекает оценка $d_{X}\left(x, M_{0}\right) \leqslant h_{1}(f(x))$, где $h_{1}(t) \rightarrow 0$ при $t \rightarrow 0$. Объединяя эту оценку с $(17)$, получаем соотношение

$$
\lim _{t \rightarrow \infty} \theta_{X}\left(U_{t}\left(M_{c}\right), M_{0}\right)=0
$$

означающее, что $M_{0}$ есть минимальный глобальный аттрактор полугруппы операторов $U_{t}: M_{c} \rightarrow M_{c}$. Включение $w \in \Lambda\left(Q \backslash M_{0}, X\right)$ и (18) влекут непрерывность полугрупшы $U_{t}$.

Последующие рассуждения аналогичны проведенным при доказательстве теоремы 1 . Пусть $\mathscr{K}_{1}\left(M_{c}\right)$ - часть $\mathscr{K}\left(M_{c}\right)$, состоящая из компактов $\mathfrak{K}$, удовлетворяюших условиям: $M_{0} \subset \mathfrak{K}, U_{t}(\mathfrak{K}) \subset \mathfrak{K}, t \geqslant 0$. На основе равенства (18) доказывается, что $\mathscr{K}_{1}\left(M_{c}\right)$ есть конфинальная часть $\mathscr{K}\left(M_{c}\right)$, рассматриваемого как направленное множество относительно порядка, порождаемого включением. Отсюда, как и выше, выводится изоморфизм групп $H_{l}\left(M_{0}\right)$ и $H_{l}\left(M_{c}\right), l=0,1, \ldots$ Согласно $(12)$ $H_{l}\left(M_{c}\right) \approx H_{l}\left(M_{1}\right)$, что и доказывает изоморфизм $H_{l}\left(M_{0}\right) \approx H_{l}\left(M_{1}\right), l=0,1, \ldots$

В частности, определены и равны между собой эйлеровы характеристики $\mathrm{X}\left(M_{0}\right), \mathrm{X}\left(M_{1}\right)$ множеств $M_{0}$ и $M_{1}$. Так как поле $\partial f$ невырождено на $M_{1} \backslash M_{0}$, то

$$
\operatorname{ind}_{Q}\left(M_{0}, \partial f\right)=\gamma_{Q}\left(\partial f, M_{1}^{0}\right)=\mathrm{X}\left(M_{1}\right)=\mathrm{X}\left(M_{0}\right) \text {. }
$$

Теорема доказана. 
Равенство $\operatorname{ind}_{Q}\left(M_{a}, \partial f\right)=\mathrm{X}\left(M_{a}\right)$ устанавливалось ранее при более жестких ограничениях на функционал $f$, пространство $X$ и множества $Q, M_{a}=\arg \min f$. Например, в [10] рассматривался функционал $f$ класса $C^{1,1}(X)$, пространство $X$ предполагалось гильбертовым, $Q=X, M_{a}$ - гладкое многообразие, расположенное в конечномерном пространстве. Порядок гладкости функционала $f$ снижен на 1 в работах [11], [14]; там же рассмотрены случаи, когда $Q \neq X$, а $M_{a}$ есть объединение конечного числа выпуклых компактов. В теореме 3 отсутствуют априорные предположения о структуре множества $M_{a}$. Из нее следует, что если $a-$ изолированное критическое (минимальное) значение функционала $f$ класса $\Lambda_{1}(Q)$, то соответствуюшее множество $M_{a}=\arg \min f$ не может быть слишком сложным, в частности имеет смысл эйлерова характеристика $\mathrm{X}\left(M_{a}\right)$. В работах [11], [14] нет аналогов первого утверждения теоремы 3.

Для некоторых функционалов изоморфизм (12) имеет место и при $b=\infty$. Функционал $f$ класса $\Lambda_{1}(Q)$ назовем коэрцитивнылм, если множество $\mathfrak{C}_{f}$ его критических точек и каждое лебегово множество $M_{t}=\{x \in Q, f(x) \leqslant t\}, t<\infty$, ограничены. В случае ограниченного множества $Q$ всякий функционал $f$ из $\Lambda_{1}(Q)$ коэрцитивен; для неограниченного множества $Q$ это, разумеется, не так. Легко доказывается компактность множества критических точек коэрцитивного функционала. Следующее утверждение обобщает некоторые результаты (см. [6, с. 66], $[11$, c. 126$],[14])$.

ТЕОРема 4. Пусть $b(f)$ - максимальное критическое значение коэрцитивного функиионала $f$. Eсли $a>b(f)$, mo $H_{l}\left(M_{a}\right) \approx H_{l}(Q), l=0,1, \ldots, u$ $\gamma\left(\partial f, M_{a}^{0}\right)=1$.

ДокАЗАТЕЛЬСтво. Пусть $b(f)<a<b<\infty$. В силу теоремы 2 множество $M_{a}$ есть деформационный ретракт множества $M_{b}$. Фиксируем целое число $l \geqslant 0$. Порождаемый вложением $i_{a}^{b}: M_{a} \rightarrow M_{b}$ гомоморфизм $\left(i_{a}^{b}\right)_{*}: H_{l}\left(M_{a}\right) \rightarrow H_{l}\left(M_{b}\right)$ является изоморфизмом. Система $\left\{H_{l}\left(M_{a}\right),\left(i_{a}^{b}\right)_{*}\right\}$ образует прямой спектр на луче $(b(f), \infty)$. Из определения групп гомологий с компактными носителями (cм. [17, c. 244], [18, с.316]) вытекает изоморфизм $H_{l}\left(M_{a}\right) \approx H_{l}(Q), l \geqslant 0$, означающий гомологическую тривиальность $\left[18\right.$, с. 43] лебеговых множеств $M_{a}$ при $a>b(f)$. Равенство $\gamma\left(\partial f, M_{a}^{0}\right)=\mathrm{X}\left(M_{a}\right)=1$ следует из теоремы 1 . Теорема доказана.

4. Обсудим возможные применения установленных выше результатов. Использование отличных от индекса топологических характеристик функционалов позволяет находить новые оценки числа критических точек и критических значений функционалов. Например, если при некотором $l \geqslant 0$ группы гомологий $H_{l}\left(M_{a}\right)$, $H_{l}\left(M_{b}\right)$ лебеговых множеств функционала $f$ не изоморфны, то, применяя теоремы 2-4, можно доказать сушествование критических значений $f$, принадлежащих отрезку $[a, b]$ (или его правильной части). Из теоремы 3 вытекает, в частности,

СЛЕДСТВИЕ. Пусть лебегово множество $M_{b}$ функционала $f$ из $\Lambda_{1}(Q)$ непусто и ограничено, $a=\min \{f(x), x \in Q\}<b$. Ecли группы $H_{l}\left(M_{a}\right)$ u $H_{l}\left(M_{b}\right)$ неизоморфны при некотором $l$, то промежуток $(a, b]$ содержит критическое 
значение $f$. Если эйлерова характеристика множества $M_{a}$ не определена, то найдется последовательность $c_{n}$ критических значений функиионала $f$, $a<c_{n} \leqslant b, \quad c_{n} \rightarrow a$.

Следствие позволяет установить существование нетривиальных (т.е. отличных от минимального) критических значений функционала. В некоторых случаях удается доказать существование бесконечного числа критических значений, а следовательно, бесконечного числа критических точек функционала $f$. Ситуация, когда эйлерова характеристика множества $\arg \min f$ не определена, в этом смысле наиболее благоприятна, вместе с тем она исключена из рассмотрения в [10], [11], [14].

Теорема 4 влечет следствие: если в условиях теоремы 4 лебегово множество $M_{c}$ гомологически нетривиально при некотором $c$, то $b(f) \geqslant c$. В частности, несвязность множества $M_{c}$ обеспечивает сушествование не меньшего, чем $c$ критического значения функционала $f$.

Рассмотрим примеры иллюстративного характера. Пусть $\mathfrak{N}$ - компакт в пространстве $X, f_{\mathfrak{N}}(x)=(1 / 2) d_{X}^{2}(x, \mathfrak{N}), x \in X$. Если $X$ и $X^{*}$-локально равномерно выпуклые пространства, то $f_{\mathfrak{N}} \in \Lambda_{1}(X)$ [14]. Очевидно, что $\mathfrak{N}=\arg \min f_{\mathfrak{N}}$ и при любом $t<\infty$ множество $\left\{x \in X, f_{\mathfrak{N}}(x) \leqslant t\right\}$ ограничено. Из результатов $[7, \S 2.8]$ вытекает равенство

$$
f_{\mathfrak{N}}^{0}(x, v)=\max \left\{\left\langle v, g^{\prime}(x-w)\right\rangle, w \in \mathfrak{N}(x)\right\},
$$

в котором $\mathfrak{N}(x)=\{w \in \mathfrak{N}, d(x, \mathfrak{N})=\|x-w\|\}$ - метрическая проекция элемента $x$ на множество $\mathfrak{N}, g(x)=\|x\|^{2} / 2$. Следствием (19) является геометрически очевидное неравенство

$$
f_{\mathfrak{N}}^{0}(x,-x)<0, \quad\|x\|>R_{0} \gg 0 .
$$

Таким образом, $f_{\mathfrak{N}}$ - коэрцитивный на $X$ функционал, и к нему применимы предшествуюшие результаты. Например, если $\mathfrak{N}$ не является гомологически тривиальным, то существует положительное критическое значение функционала $f_{\mathfrak{N}}$; если эйлерова характеристика компакта $\mathfrak{N}$ не определена, то имеется последовательность $c_{n}$ критических значений функционала $f_{\mathfrak{N}}: c_{n}>0, c_{n} \rightarrow 0$. Если $X$-гильбертово пространство, $\mathfrak{N}$ - невыпуклый компакт, то функционал $f_{\mathfrak{N}}$ не является ни гладким, ни выпуклым.

Изложенная схема обнаружения нетривиальных критических значений может быть распространена на широкий класс функционалов. Полезно заметить, что интегральные функционалы, рассматриваемые на подходящем пространстве Соболева, в естественных предположениях принадлежат классу $\Lambda_{1}$; соответствующие примеры гладких функционалов можно найти в [2], [10], [16], [24]. Отказ от гладкости позволяет расширить множество подобных функционалов. Из результатов работы [25] вытекает

ПРЕДЛОЖЕНИЕ 2. Пусть $\omega$ - ограниченная область в $\mathbb{R}^{m}$, функиия $\Phi: \mathbb{R}^{k} \rightarrow \mathbb{R}$ строго выпукла и удовлетворяет неравенствам $c_{1}|\xi|^{p} \leqslant \Phi(\xi) \leqslant$ $c_{2}|\xi|^{p}$, в которых $|\xi| \geqslant 1, \quad 0<c_{1}<c_{2}<\infty, \quad p>1$. Тогда функционал

$$
I_{\Phi}(y)=\int_{\omega} \Phi(y(x)) d x
$$


принадлежит классу $\Lambda_{1}\left(L_{p}\left(\omega, \mathbb{R}^{k}\right)\right)$.

В условиях предложения 2 функция $\Phi: \mathbb{R}^{k} \rightarrow \mathbb{R}$ и функционал $I_{\Phi}$ : $L_{p}\left(\omega, \mathbb{R}^{k}\right) \rightarrow \mathbb{R}$ могут быть негладкими; в качестве примера достаточно рассмотреть функцию $\Phi(\xi)=|\xi|^{p}+|\xi|$. Такого рода функции характерны для вариационных задач, возникающих в механике вязкопластических сред [26].

Соответствуюшие примеры несложно привести и для пространства Соболева $\stackrel{\circ}{W}_{p}^{1}(\omega)$ функций $u: \omega \rightarrow \mathbb{R}$, градиенты $\nabla u$ которых принадлежат пространству $L_{p}\left(\omega, \mathbb{R}^{m}\right)$, а следы на $\partial \omega$ равны нулю. В частности, функционал $f(u)=I_{\Phi}(\nabla u)+$ $f_{1}(u)$ принадлежит $\Lambda_{1}(\stackrel{\circ}{W} \underset{p}{1}(\omega))$, если функция $\Phi: \mathbb{R}^{m} \rightarrow \mathbb{R}$ удовлетворяет условиям предложения 2, а $f_{1}: \stackrel{\circ}{W} p(\omega) \rightarrow \mathbb{R}$ - гладкий функционал, производная $f_{1}^{\prime}$ которого есть вполне непрерывный оператор из пространства $\stackrel{\circ}{W} \underset{p}{1}(\omega)$ в сопряженное к нему. В данном примере функционал $f$ имеет смешанную гладко выпуклую структуру, что типично для функционалов вариационного исчисления.

\section{Список литературы}

1. Похожсаев С.И. О разрешимости нелинейных уравнений с нечетными операторами // Функцион. анализ и его прилож. 1967. Т. 1. № 3. С. 66-73.

2. Скрыпник И. В. Методы исследования нелинейных эллиптических граничных задач. М.: Наука, 1990.

3. Browder F. E. Nonlinear elliptic boundary value problems and the generalized topological degree // Bull. Amer. Math. Soc. 1970. V. 76. № 5. P. 999-1005.

4. Борисович Ю. Г. Об относительном вращении компактных векторных полей в линейных пространствах // Тр. семин. по функц. анализу. Вып. 12. Воронеж: Изд-во Воронежского ун-та, 1969. С. 3-27.

5. Борисович Ю. Г., Гельман Б. Д., Мьчкис А. Д., Обуховский В. В. Топологические методы в теории неподвижных точек многозначных отображений // УМН. 1980. Т. 35. № 1. C. $59-126$.

6. Красносельский М. А., Забрейко П. П. Геометрические методы нелинейного анализа. М.: Наука, 1975.

7. Кларк . Оптимизация и негладкий анализ. М.: Наука, 1988.

8. Милнор Дж., Уоллес А. Дифференциальная топология. Начальный курс. М.: Мир, 1972.

9. Иоффе А. Д., Тихомиров В. М. Двойственность выпуклых функций и экстремальные задачи // УМН. 1968. Т. 23. №6. С. 51-116.

10. Бобылев Н. А. О топологическом индексе экстремалей многомерных вариационных задач // Функцион. анализ и его прилож. 1986. Т. 20. № 2. С. 8-13.

11. Бобылев H. А., Климов В. С. Методы нелинейного анализа в задачах негладкой оптимизации. М.: Наука, 1992.

12. Заславский $A$. Я. О критических точках липшицевых функций на гладких многообразияx // СMЖ. 1981. Т. 22. №1. C. 87-93.

13. Климов В. С. Минимаксные критические значения негладких функционалов // СМЖ. 1992. T. 33. № 3. C. 91-100.

14. Климов В.C., Сенчакова Н. В. Об относительном вращении многозначных потенциальных векторных полей // Матем. сб. 1991. Т. 182. № 10. С. 1393-1407.

15. Сенчакова Н.В. Вращение многозначного векторного поля и решения вариационных неравенств // Качественные методы исследования операторных уравнений. Ярославль: Изд-во Яросл. ун-та, 1988. С. 70-78. 
16. Скрыпник И. В. Нелинейные эллиптические уравнения высшего порядка. Киев: Наук. думка, 1973.

17. Масси У. Теория гомологий и когомологий. М.: Мир, 1981.

18. Стинрод Н., Эйленберг С. Основания алгебраической топологии. М.: ИЛ, 1958.

19. Лионс ЖК. Л. Некоторые методы решения нелинейных краевых задач. М.: Мир, 1972.

20. Дмитрук А.В., Милютин А. А., Осмоловский Н. П. Теорема Люстерника и теория экстремума // УМН. 1980. Т. 35. №6. С. 11-46.

21. Dold A. Fixed point and fixed point theorem for Euclidean neighbourhood retracts // Topology. 1965. V. 4. P. 1-8.

22. Дольд А. Лекции по алгебраической топологии. М.: Мир, 1976.

23. Лады женская $O . A$. О нахождении минимальных глобальных аттракторов для уравнений Навье-Стокса и других уравнений с частными производными // УМН. 1987. Т. 42. №6. C. $26-60$.

24. Климов В. С. О числе решений краевых задач нелинейной теории оболочек // ДАН CCCP. 1979. T. 241. № 1. C. 57-60.

25. Климов $B$. C. Почти периодические решения эволюционных задач механики вязкопластических сред // Изв. ВУЗов. Сер. Математика. 1994. №1. С. 17-24.

26. Мосолов П. П., Мясников В. П. Механика жесткопластических сред. М.: Наука, 1981.

Орловский государственный университет

Поступило в редакцию

3.XII.1996 